\title{
Características de refugios de algunas especies de murciélagos en la cuenca alta del rio Itaya, Loreto, Perú
}

\author{
Edgardo M. Rengifo ${ }^{1}$, Wendy Calderón ${ }^{1}$ y Rolando Aquino ${ }^{2}$ \\ 1. Museo de Historia Natural de la Universidad Nacional Mayor de San Marcos, Apartado 14-0434, Lima-14, Perú; \\ edgar_mrv@hotmail.com,wcalderonsv@yahoo.es \\ 2. Instituto de Ciencias Biológicas Antonio Raimondi, Facultad de Ciencias Biológicas, Universidad Nacional Mayor de San Marcos. P. O. Box 575 \\ Iquitos, Perú; raquinoy2005@yahoo.es
}

Recibido 30-VIII-2012 Corregido 28-X-2012 Aceptado 23-XI-2012

\begin{abstract}
Roost characteristics for some bat species of the Itaya river headwaters, Loreto, Peru. This study presents information about bat roosts and their users in the Itaya River headwater, approximately $80 \mathrm{~km}$ Southwest of Iquitos City (Northeastern Peru). This study was made from May to October of 2008. We established four sampling localities which were evaluated through intensive searches of potential roosts in line transects. The sampling effort covered $98 \mathrm{~km}$ and we recorded 21 roosts corresponding to six types. The most common roost type was the tree cavity ( $n=12)$, followed by bark ( $n=5)$, furled leaf $(n=1)$, termite nest $(n=1)$, tent $(n=1)$ and eroded dirt $(n=1)$ : this last type is described here for the first time. We identified 14 species representing 12 genera and 4 families (Phyllostomidae, Thyropteridae, Furipteridae and Emballonuridae). Roosts had 1-3 species, but most harbored only one. Phyllostomidae (except Choeroniscus minor), Thyropteridae and Furipteridae used protected sites such as tree cavities, termite nests, furled leaves and tents, while Emballonuridae were often found in exposed sites.
\end{abstract}

KEY WORDS

Roosts, bats, Itaya River, Loreto, Peru.

\section{RESUMEN}

El presente estudio proporciona información sobre los refugios de murciélagos de la cuenca alta del río Itaya, localizado aproximadamente a $80 \mathrm{~km}$ del sureste de la ciudad de lquitos (Nororiente de Perú). Este estudio fue realizado desde mayo a octubre del 2008, donde evaluamos cuatro localidades, realizando búsquedas intensivas de refugios mediante caminatas diurnas. Recorrimos un total de $98 \mathrm{~km}$, registramos 21 refugios; el tipo más común fue la cavidad de árbol $(n=12)$ seguido de corteza de árbol $(n=5)$, hoja enrollada $(n=1)$, termitero $(n=1)$, tienda $(n=1)$ y erosión de tierra $(n=1)$. Registramos 14 especies de murciélagos siendo usuarias de los refugios, las cuales representaron a 12 géneros y 4 familias (Phyllostomidae, Thyropteridae, Furipteridae y Emballonuridae). Los registros variaron de una a tres especies por refugio, siendo en su mayoría aquellas habitadas por una sola especie. Las especies de la familia Phyllostomidae (a excepción de Choeroniscus minor), Thyropteridae y Furipteridae usaron refugios no expuestos como cavidades de árboles, termiteros, hojas tiernas de heliconias y tiendas; mientras las especies de la familia Emballonuridae, fueron encontrados en su mayoría en lugares expuestos.

\section{PALABRAS CLAVE}

Refugios, murciélagos, Rio Itaya, Loreto, Perú.
Los murciélagos, con más de 1100 especies descritas actualmente, habitan en casi todos los hábitats del mundo (Simmons, 2005); una de las características funcionales que les ha ofrecido ventajas de dispersión y colonización frente a otros mamíferos ha sido la capacidad de vuelo (Fleming \& Eby, 2003), por lo que han tenido que adaptarse, entre otras necesidades, a gran variedad de refugios como árboles, hojas, nidos de aves, termiteros, cavernas, grietas de rocas, minas, puentes, edificios, entre otras estructuras (Kunz
\& Lumsden, 2003). Los refugios proporcionan muchos beneficios, como la reducción de competencia entre especies simpátricas, ahorro de energía para su metabolismo, sitios de descanso, protección contra el clima y depredadores (Fenton et al.,2000); además permite una compleja interacción de respuestas fisiológicas, etológicas y adaptativas, influyendo en la organización social, distribución y en la abundancia de recursos alimenticos (Kunz, 1982; Aguirre, Lens \& Matthysen, 2003). 
El Perú es uno de los países con mayor diversidad de murciélagos en el mundo (Pacheco et al., 2009; Lim et al., 2010; Velazco \& Cadenillas, 2011), sin embargo los estudios en ecología aún son escasos, y más aún trabajos enfocados a refugios de murciélagos (e.g., Graham 1988, Simmons, Voss \& Fleck, 2002; Fleck, Voss \& Simmons, 2002; Tello \& Velazco, 2003; Hice, Velazco \& Willig, 2004), debido principalmente a que los métodos usados para el registro y estudio de murciélagos están basados casi exclusivamente en la captura con redes de neblina, pese a la existencia de diversos métodos (Tuttle, 1976); uno de ellos es la búsqueda de refugios, método que ofrece mayor probabilidad de registro de especies que son difíciles de capturar en redes de neblina o de especies que tienen preferencia por los estratos arbóreos (Voss \& Emmons, 1996).

Una de las regiones dentro del territorio peruano con la más alta riqueza de especies es Loreto (Ascorra \& Wilson, 1992; Ascorra, Gorchov \& Conejo, 1993; Fleck et al., 2002; Hice et al., 2004), sin embargo muchas áreas de esta región no han sido exploradas como es el caso de la cuenca alta del río Itaya, en la que no existen estudios previos sobre la diversidad de murciélagos, siendo los inventarios más cercanos correspondientes a la Reserva Nacional Allpahuayo-Mishana (López-Wong, 2002; Hice et al., 2004) y alrededores (Davis \& Dixon, 1976).

El presente estudio proporciona información sobre refugios de murciélagos, en base a trabajos de campo realizados en cuatro localidades de muestreo de la cuenca alta del río Itaya en Loreto, Perú, con el objetivo de contribuir al conocimiento de los tipos de refugios y a las especies de murciélagos que las ocupan.

\section{METODOLOGIA}

El estudio fue llevado a cabo en la cuenca alta del río Itaya, la cual se ubica entre los ríos Amazonas, Marañón y Nanay, aproximadamente $80 \mathrm{~km}$ al suroeste de la ciudad de lquitos, en el centro de una de las eco regiones con alta prioridad de conservación a nivel regional por su excepcional biodiversidad (Dinerstein et al., 1995) denominada "Eco-región Bosques Húmedos del Napo" o "Eco-región Napo". Esta zona se caracteriza por presentar una fisiografía variada donde predominan las terrazas y colinas (Malleux, 1982), con pendientes de $40-60^{\circ}$ de inclinación (Aquino et al., 2007; 2009), cobertura vegetal compuesta principalmente por árboles como Parkia nitida, Elaeoluma sp., Manilkara sp. y Eschweilera sp., que miden entre 10 a $25 \mathrm{~m}$ y algunos árboles emergentes por encima de los $30 \mathrm{~m}$, además el sotobosque es relativamente abierto, dominado por Lepidocaryum tenue (Aquino et al., 2008). En este estudio fueron identificados tres tipos de hábitat diferentes según la clasificación de Encarnación (1993): Bosque de colina baja moderadamente disectada (BCb$\mathrm{md}$ ), Bosque de terraza baja (BTb) y Bosque de colina baja fuertemente disectada (BCb-fd).

Los trabajos de campo se desarrollaron de mayo a octubre del 2008 abarcando las dos estaciones presentes en la Amazonía, vaciante y creciente (Sioli, 1984). Cuatro localidades de muestreo fueron evaluados (Fig. 1): Nauta (BCb-md) (4ำ $\left.15^{\prime} 21^{\prime \prime} \mathrm{S}, 73^{\circ} 51^{\prime} 56^{\prime \prime} \mathrm{W}\right)$; Agua Blanquillo (BTb) $\left(4^{\circ} 12^{\prime} 0,1^{\prime \prime} \mathrm{S}, 73^{\circ} 52^{\prime} 8,5^{\prime \prime} \mathrm{W}\right)$; Seis Unidos (BCb-fd) $\left(04^{\circ} 12^{\prime} 6^{\prime \prime} \mathrm{S}\right.$,

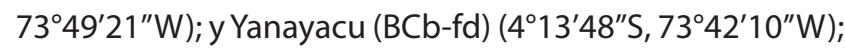
en cada localidad de muestreo se establecieron cinco transectos lineales de dos kilómetros de longitud.

Para la búsqueda de refugios se recorrieron los transectos lineales a un ritmo de $1 \mathrm{~km} / \mathrm{hora}$, el recorrido se hizo a ambos lados de los transectos de ida y vuelta, haciendo un total de 2 a $3 \mathrm{~km}$ por día, la búsqueda se hizo básicamente al nivel del suelo entre $0-5 \mathrm{~m}$ de altura, observando detalladamente los lugares potenciales como: cavidades en árboles, hojas de Musaceas y Arecaceas, cavernas, entre otros (Graham, 1988; Fenton et al., 2000; Kalko, Veberschaer \& Dechmann, 2006); después de ubicar estos potenciales refugios se verificó la presencia de murciélagos, para este propósito se usó varillas delgadas y linternas para perturbar el lugar, luego, confirmada la presencia de murciélagos en el refugio, se procedió a colocar una red de neblina en los alrededores, posteriormente mediante hostigamiento se esperó la salida de los individuos, quedando éstos atrapados en la red. Cuando no fue posible la captura de los individuos se tomó nota de la especie observada siempre y cuando haya sido identificado hasta nivel de especie. Para la clasificación de los refugiosse siguió el criterio de Kunz y Lumsden (2003).

\section{RESULTADOS}

El esfuerzo de muestreo empleado fue de $98 \mathrm{~km}$ recorridos durante 40 días de evaluación efectiva. Se encontraron 21 refugios correspondientes a seis tipos, cinco de los cuales corresponden a la clasificación de Kunz \& Lumsden (2003) y otro a un nuevo tipo de refugio, el cual lo denominamos: erosión de tierra. En estos refugios se registraron 14 especies de murciélagos pertenecientes a 12 géneros y 4 familias.

A continuación se hace una breve descripción de los tipos de refugios encontrados y las especies registradas, se menciona el nombre científico de la planta hospedera y entre comillas el nombre local, el número entre corchetes indica el registro según el Cuadro 1. 


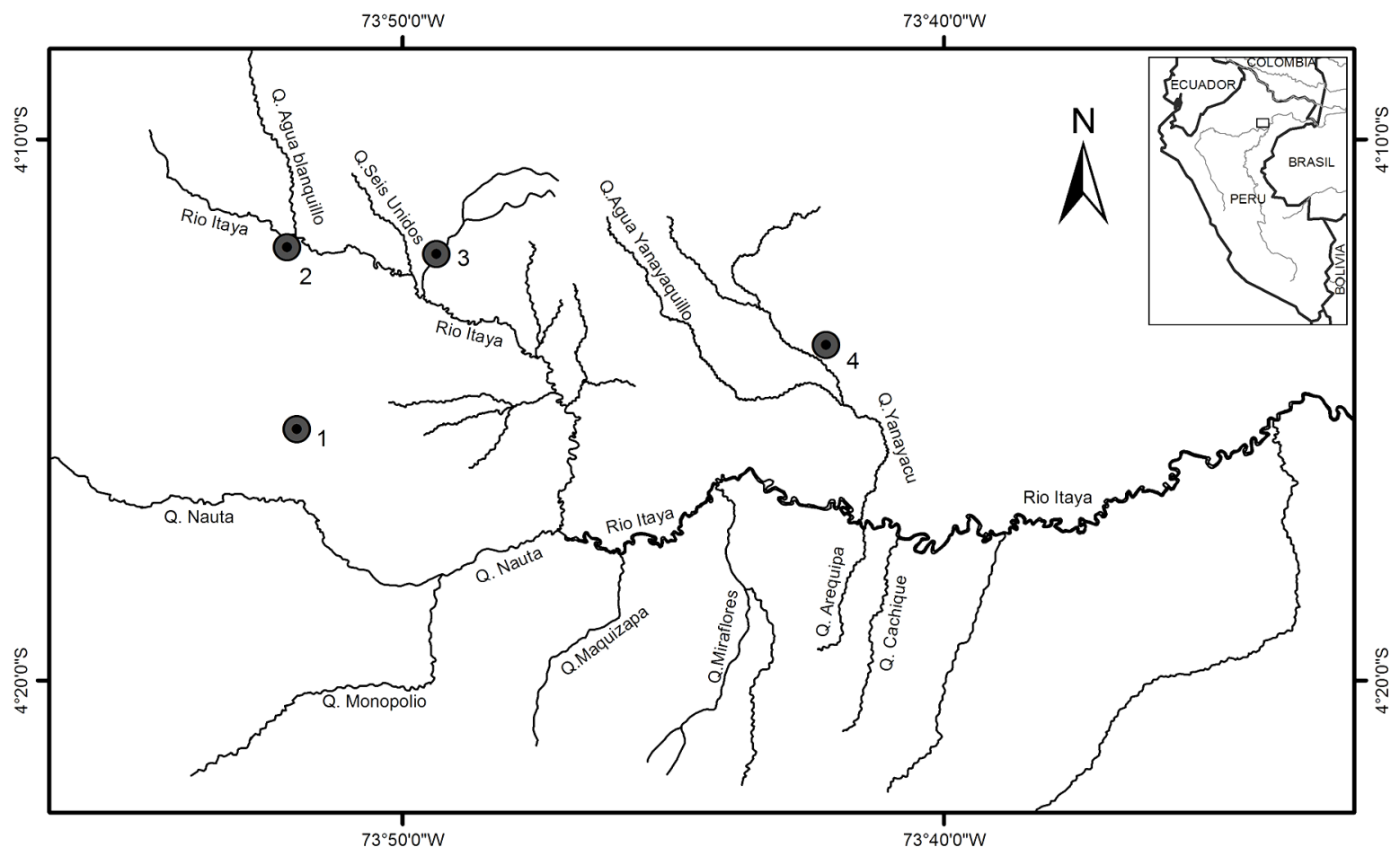

FIG. 1. Localidades de muestreo en la cuenca alta del rio Itaya: 1 = Nauta. $2=$ Agua Blanquillo. $3=$ Seis Unidos.4=Yanayacu.

\section{Cavidad de árbol}

Fue el más común, 12 de los refugios corresponden a este tipo; entre los diferentes refugios de este tipo observamos que la ubicación de las cavidades varió en diferentes partes del árbol, siendo las más comunes entre raíces y parte central de árboles caídos y algunos pocos en cavidades formadas en árboles en pie, estos refugios se caracterizaron por no estar expuestos, ya que se encontraban protegidos de las Iluvias manteniendo un ambiente relativamente seco, aunque los refugios ubicados en raíces de árboles caídos solo eran protegidos por las raíces o tierra.

Nueve especies de murciélagos, cinco de la familia Phyllostomidae, tres de la familia Emballonuridae y uno de la familia Furipteridae fueron registrados. En cavidades formadas en las raíces de árboles caídos fueron registrados individuos solitarios correspondientes a Peropteryx macrotis [20] y Furipterus horrens [17], mientras que Micronycteris megalotis $[10,15]$ en pareja. En cavidades formadas en el centro de árboles caídos, fueron registrados individuos solitarios de Peropteryx leucoptera [2, 21], mientras que en cavidades más grandes de árboles caídos se observaron varias especies compartiendo el mismo refugio, dichas asociaciones estuvieron conformadas por Micronycteris megalotis + Carollia perspicillata + Carollia brevicauda [11] y por Carollia perspicillata + Saccopteryx bilineata [12]. En las cavidades formadas en el centro de árboles en pie se registraron a Micronycteris minuta [3] y Micronycteris megalotis [9] en parejas, mientras que en refugios donde la cavidad comprometía gran parte del árbol, se observaron asociaciones como Carollia perspicillata + Glossophaga soricina + Saccopteryx bilineata [7] y Carollia perspicillata + Micronycteris megalotis [14].

\section{Corteza de árbol}

Fueron encontrados cinco refugios de este tipo, registrándose tres especies de la familia Emballonuridae y una especie de la familia Phyllostomidae. Representando a este tipo de refugio encontramos: un árbol caído correspondiente a Eschweilera sp., albergando un individuo de Peropteryx leucoptera [4], el cual estuvo perchado debajo del tronco a un metro del suelo; un árbol en pie, también 
de Eschweilera sp., donde se registró una colonia de aproximadamente 20 individuos de Saccopteryx bilineata [18]; un árbol inclinado de Ficus sp. donde se reportó un grupo de seis individuos de Rhynchonycteris naso [8], y dos árboles caídos, posicionados en ángulo horizontal, donde se encontró en ambos casos parejas de Choeroniscus minor $[16,19]$ perchados debajo del árbol.

\section{Hoja enrollada}

Este refugio fue registrado en una sola ocasión, la especie vegetal hospedera fue una hoja tierna de Heliconia sp. de aproximadamente 1 metro de altura que se encontraba enrollada en forma de cono. Se registró un individuo de la familia Thyropteridae, Thyroptera tricolor [6], el cual se encontraba adherido a la hoja con ayuda de sus ventosas.

\section{Termitero}

Este tipo de refugio fue encontrado cubriendo la zona del capitel de la palmera Astrocaryum huicungo "Huicungo" a 1,8m del suelo. El termitero se encontraba activo, y tenía una medida de $51 \mathrm{~cm}$ de alto, $42 \mathrm{~cm}$ de ancho y una profundidad de $32 \mathrm{~cm}$, presentaba un orificio de entrada en la parte inferior, el cual estaba protegido por las espinas de la palmera ante posibles ataques de depredadores. Cinco individuos de Lophostoma silvicolum [13] fueron observados dentro del refugio, siendo capturado solo un individuo, no fue posible determinar la edad ni el sexo de los demás miembros del grupo.

\section{Tienda}

Este tipo de refugio fue encontrado en una sola ocasión, siendo la palmera Lepidocaryum tenue "Irapay" la especie vegetal hospedera, se observó la punta de la hoja modificada, debido a mordeduras en las nervaduras producidas por el murciélago, el tipo de tienda corresponde a la arquitectura tipo Apical según Rodríguez-Herrera, Medellín \& Timm (2007). Un solo individuo de la familia Phyllostomidae fue registrada, Mesophylla macconnelli [1] perchado en la hoja de $L$. tenue.

\section{Erosión de tierra (Land erosion)}

Solo se encontró un refugio de este tipo, el cual estaba localizado en la ladera de una colina con pendiente de $30^{\circ}$ a $35^{\circ}$ de inclinación; la erosión tenía una profundidad aproximada de $2,5 \mathrm{~m}, 3 \mathrm{~m}$ de ancho y $3,5 \mathrm{~m}$ de largo, además de la presencia de pequeñas raíces sobresalientes provenientes de árboles y arbustos situados en la periferia, en general la erosión tenia forma de zanja semicircular, otra característica importante del lugar fue el buen drenaje del suelo, lo cual podría haberse originado por algún proceso de percolación. En este refugio solo se registró un individuo de Peropteryx leucoptera [5], el cual fue observado perchado en una de las raíces sobresalientes.

\section{DISCUSIÓN}

La búsqueda de refugios es una práctica de registro poco difundida en los inventarios llevados a cabo en la Amazonía; el registro de 14 especies en este estudio, usando este método, corroboran su alta eficacia sugerida anteriormente por Voss \& Emmons (1996), especialmente en aquellas especies difíciles de capturar en redes de neblina; López-Wong (2002) en Allpahuayo - Mishana menciona que ocho de las 30 especies que reportó fueron registradas en refugios, del mismo modo Hice et al. (2004) quien realizó búsquedas con un esfuerzo de muestreo de aproximadamente 25 horas registró 10 especies de murciélagos, además encuentra 17 especies en un alcantarillado debajo de la Carretera Iquitos-Nauta, en ambos estudios los refugios encontrados no son descritos en detalle. Cabe resaltar que nuestro estudio estuvo enfocado básicamente a nivel del sotobosque, de este modo aquellos refugios que se encontraban en el dosel quedaron sin ser observados, debido a lo difícil que es ubicar refugios en las partes altas del bosque, por lo que consideramos que aún existe un sesgo de información.

De los seis tipos de refugios mencionados en este estudio, la cavidad de árbol fue el más común; el mayor uso por murciélagos de éste se ha sugerido que se debe a la protección que ofrece frente a depredadores, factores ambientales y climáticos como temperatura, humedad, precipitaciones y vientos, creando así un ambiente óptimo para el refugio de murciélagos (Bradbury, 1977; Kunz, 1982), tres familias fueron usuarias de este tipo de refugio: Phyllostomidae, Emballonuridae y Furipteridae, de las cuales la más frecuente fue la familia Phyllostomidae, confirmando así lo mencionado por Tuttle (1976) y Fenton et al., 2001, quienes señalan que en el Neotrópico las cavidades de árboles son usadas principalmente por las especies de esta familia. En el tipo de refugio hoja enrollada se registró a Thyroptera tricolor lo cual está relacionado a las adaptaciones de esta especie, pues ha desarrollado sobre el pulgar y pata trasera discos de succión que le permite adherirse a superficies (Carvalho, 1940), permitiéndole de este modo protegerse de los factores externos. En el refugio tipo termitero solo se registra a Lophostoma silvicolum, refugio que podría proporcionar beneficios contra lluvia, depredadores y reducción de ectoparásitos (Kalko et al., 2006). En el refugio tipo tienda fue encontrado solo Mesophylla macconnelli, este tipo de refugio según 
CUADRO 1

Lista de los refugios registrados en la cuenca alta del rio Itaya

\begin{tabular}{|c|c|c|c|c|c|}
\hline $\mathbf{N}^{\circ}$ & $\begin{array}{l}\text { Fecha de } \\
\text { registro }\end{array}$ & Tipo de refugio & $\begin{array}{l}\text { Especie hospedera } \\
\text { (Nombre local) }\end{array}$ & $\begin{array}{c}\text { Hábitat } \\
\text { (Localidad) }\end{array}$ & Especie de murciélago (sexo) \\
\hline 1 & $25 / 05 / 2008$ & Tienda & $\begin{array}{l}\text { Lepidocaryum tenue } \\
\text { (Irapay) }\end{array}$ & $\mathrm{BCb}-\mathrm{md}(1)$ & Mesophylla macconnelli* \\
\hline 2 & $26 / 05 / 2008$ & Cavidad de árbol & $?$ & $\mathrm{BCb}-\mathrm{md}(1)$ & Peropteryx leucoptera (ㅇ) \\
\hline 3 & $26 / 05 / 2008$ & Cavidad de árbol & $\begin{array}{l}\text { Tachigali sp. } \\
\text { (Tangarana) }\end{array}$ & $\mathrm{BCb}-\mathrm{md}(1)$ & Micronycteris minuta (3 $\left.\sigma^{7}\right)$ \\
\hline 4 & $27 / 05 / 2008$ & Corteza & $\begin{array}{l}\text { Eschweilera sp. } \\
\text { (Machimango) }\end{array}$ & $\mathrm{BCb}-\mathrm{md}(1)$ & Peropteryx leucoptera (o') \\
\hline 5 & $27 / 05 / 2008$ & Erosión de tierra & $?$ & $\mathrm{BCb}-\mathrm{md}(1)$ & Peropteryx leucoptera (ơ⿱) \\
\hline 6 & $28 / 05 / 2008$ & Hoja enrollada & Heliconia sp. (Heliconia) & $\mathrm{BCb}-\mathrm{md}(1)$ & Thyroptera tricolor ( $\left(^{\top}\right)$ \\
\hline 7 & $28 / 05 / 2008$ & Cavidad de árbol & $\begin{array}{l}\text { Minquartia guianensis } \\
\text { (Huacapu) }\end{array}$ & $\mathrm{BCb}-\mathrm{md}(1)$ & $\begin{array}{l}\text { Saccopteryx biliniata }\left(0^{7}\right) \\
\text { Glossophaga soricina }\left(0^{7}\right) \text {; } \\
\text { Carollia perspicillata }(2+)\end{array}$ \\
\hline 8 & 01/06/2008 & Corteza & Ficus sp. (Ficus) & B-tb (2) & Rhynchonycteris naso* \\
\hline 9 & $02 / 06 / 2008$ & Cavidad de árbol & Slonea sp. (Cepachina) & B-tb (2) & Micronycteris megalotis $\left(\right.$ ( , $\left.\mathrm{o}^{7}\right)$ \\
\hline 10 & $09 / 06 / 2008$ & Cavidad de árbol & $?$ & $\mathrm{BCb}-\mathrm{fd}(3)$ & Micronycteris megalotis $\left(\right.$ (, $\left.\mathrm{o}^{\top}\right)$ \\
\hline 11 & 09/06/2008 & Cavidad de árbol & $\begin{array}{l}\text { Cedrelinga } \\
\text { catenaeformis } \\
\text { (Tornillo) }\end{array}$ & $\mathrm{BCb}-\mathrm{fd}(3)$ & $\begin{array}{l}\text { Carollia perspicillata }\left(0^{7}\right) ; \\
\text { Carollia brevicauda }\left(20^{7}\right) ; \\
\text { Micronycteris megalotis ( }\left(^{7}\right)\end{array}$ \\
\hline 12 & $14 / 06 / 2008$ & Cavidad de árbol & $\begin{array}{l}\text { Pleurothyrium sp. } \\
\text { (Añuje rumo) }\end{array}$ & $\mathrm{BCb}-\mathrm{fd}(4)$ & $\begin{array}{l}\text { Saccopteryx biliniata (o); } \\
\text { Carollia perspicillata }\left(0^{7}\right)\end{array}$ \\
\hline 13 & $14 / 06 / 2008$ & Termitero & $\begin{array}{l}\text { Astrocaryum huicungo } \\
\text { (Huicungo) }\end{array}$ & $\mathrm{BCb}-\mathrm{fd}(4)$ & Lophostoma silvicolum () \\
\hline 14 & $20 / 07 / 2008$ & Cavidad de árbol & $?$ & $\mathrm{BCb}-\mathrm{fd}(3)$ & $\begin{array}{l}\text { Carollia brevicauda (\$); } \\
\left.\text { Micronycteris megalotis (( }{ }^{\top}\right)\end{array}$ \\
\hline 15 & $20 / 07 / 2008$ & Cavidad de árbol & $?$ & $\mathrm{BCb}-\mathrm{fd}(3)$ & Micronycteris megalotis (20 $)$ \\
\hline 16 & $22 / 07 / 2008$ & Corteza & Virola sp. (Cumala) & $\mathrm{BCb}-\mathrm{fd}(3)$ & 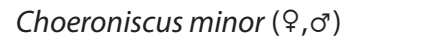 \\
\hline 17 & $28 / 07 / 2008$ & Cavidad de árbol & Ficus sp. (Renaco) & $\mathrm{BCb}-\mathrm{fd}(4)$ & Furipterus horrens (우) \\
\hline 18 & $28 / 07 / 2008$ & Corteza & $\begin{array}{l}\text { Eschweilera sp. } \\
\text { (Machimango) }\end{array}$ & $\mathrm{BCb}-\mathrm{fd}(4)$ & Saccopteryx biliniata $\left(5 \%, 40^{7}\right)$ \\
\hline 19 & $29 / 07 / 2008$ & Corteza & $\begin{array}{l}\text { Iryanthera sp. } \\
\text { (Cumala colorada) }\end{array}$ & $\mathrm{BCb}-\mathrm{fd}(4)$ & 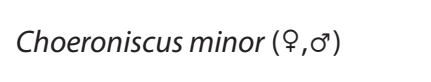 \\
\hline 20 & $29 / 07 / 2008$ & Cavidad de árbol & $?$ & $\mathrm{BCb}-\mathrm{fd}(4)$ & 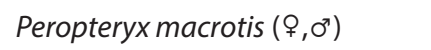 \\
\hline 21 & 06/10/2008 & Cavidad de árbol & $?$ & $\mathrm{BCb}-\mathrm{md}(1)$ & Peropteryx leucoptera (o') \\
\hline
\end{tabular}

Especie vegetal hospedera no identificada (?).

Localidades de muestreo: Nauta (1), Agua blanquillo (2), Seis Unidos (3), Yanayacu (4).

Especies no capturadas pero si identificadas mediante observación (*). Macho (ơ ) Hembra (\$). 
Foster (1992) le daría protección contra posibles depredadores. Respecto al refugio tipo Corteza, fueron registrados tanto especies de la familia Emballonuridae como una de Phyllostomidae (Choeroniscus minor), lo que caracterizó a este tipo de refugio fue la inclinación de los árboles, observado también por Kunz \& Lumsden (2003), condición que impide que la lluvia afecten a los murciélagos que usan estos árboles como refugios. En cuanto al refugio tipo Erosión de tierra con una sola especie registrada, Peropteryx leucoptera, también se caracterizó por estar expuesto. De este modo observamos que los miembros de la familia Emballonuridae fueron encontrados tanto en lugares protegidos como expuestos, tal comportamiento también fue observado por Voss \& Emmons (1996) y Simmons \& Voss (1998), quienes mencionan que las especies de esta familia ocupan las cortezas de los árboles como lugar de descanso; en tanto que Kunz (1982) sostiene que el tipo de postura y las líneas faciales y dorsales presentes en estas especies son factores de protección ante posibles depredadores, por lo que el uso de lugares expuestos no es peligroso para estos murciélagos.

Los resultados aquí presentados, coinciden con estudios previos; así, las especies Peropteryx leucoptera y Peropteryx macrotis fueron encontradas anteriormente en corteza debajo de árboles caídos y cavidades de árboles (Sanborn, 1937; Lemke et al., 1982; Brosset \& CharlesDominique, 1991; Simmons \& Voss, 1998), pero no existen registros anteriores de que alguna de estas especies ocupan algún tipo de refugio similar a la erosión de tierra, aunque Gaisler (1979) menciona que esto podría suceder, éste constituye el primer registro de este tipo; Saccopteryx bilineata con frecuencia es observada perchando en cortezas y en cavidades de árboles formando grupos grandes (Bradbury \& Emmons, 1974; Simmons \& Voss, 1998) en este estudio se registró a esta especie tanto en cavidades de árboles como en corteza; Rhynchonycteris naso, al igual que nuestras observaciones, es comúnmente registrada cerca a quebradas o ríos pequeños perchando en cortezas de árboles formando grupos de 6 a 30 individuos (Bradbury \& Emmons 1974, Simmons \& Voss 1998); Choeroniscus minor fue encontrada por Simmons y Voss (1998) perchando en cortezas debajo de árboles caídos similar a nuestros registros; en referencia a Glossophaga soricina, Micronycteris megalotis, Micronycteris minuta, $\mathrm{Ca}$ rollia brevicauda y Carollia perspicillata, han sido encontradas en diversos tipos refugios como cavernas, cavidades de árboles, minas, edificios, debajo de pozos y puentes, sin embargo, estas mismas especies en los bosques Amazónicos fueron encontradas mayormente en cavidades de árboles (Graham, 1988; Sanborn, 1949; Goodwin \& Greenhall, 1961; Handley, 1976; Greenhall \& Paradiso, 1968; Simmons \& Voss, 1998; Hice et al., 2004) en este estudio estas fueron registradas solo en cavidades de árboles; por su parte Lophostoma silvicolum ha sido estudiada por varios investigadores (Sanborn, 1951; Tuttle, 1970; Handley, 1976; McCarthy, Cadena \& Lemke, 1983; Giannini \& Kalko, 2004; Kalko et al., 2006; Dechmann, Kalko \& Kerth, 2004; Dechmann, Onig \& Kerth, 2005), quienes mencionan que esta especie modifica y usa los termiteros como refugios, de igual manera esta especie es reportada en este estudio usando este tipo de refugio; Mesophylla macconnelli construye tiendas en diversas palmeras como Geonoma sp., Anthurium sp., Astrocaryum sp. (Handley, 1976; Koepcke, 1984; Foster, 1992), pero ninguno hace referencia a Lepidocaryum tenue, especie vegetal que es muy habitual en la cuenca alta del río Itaya (Aquino et al., 2008; 2009); Furipterus horrens ha sido reportada formando grupos de hasta 250 individuos en cuevas (LaVal \& Rodriguez, 2002), sin embargo en este estudio fue encontrado solo un individuo en una cavidad de árbol caído, coincidiendo con los registros de Simmons \& Voss (1998), quienes encontraron individuos solitarios en cavidades de árboles caídos; Thyroptera tricolor usa como refugio hojas tiernas de algunas especies del orden Zingiberales (Carvalho, 1940; Simmons \& Voss, 1998; LaVal \& Rodriguez, 2002), comportamiento observado en este estudio al usar como refugio a Heliconia sp. En tal sentido todos los murciélagos reportados en este estudio, según la clasificación de Gaisler (1979), corresponden a especies tipo Fitófilos, pues en todos los casos las encontramos usando como refugio parte de especies vegetales.

La mayoría de refugios fueron encontrados con una sola especie, sin embargo, también registramos refugios habitados hasta por tres especies. Esta asociación fue observada entre las especies Saccopteryx bilineata, Glossophaga soricina, Micronycteris megalotis, Carollia perspicillata y Carollia brevicauda en el refugio tipo cavidad de árbol, donde se los reporta compartiendo el mismo refugio. Este comportamiento respondería a la interacción intra-específica positiva mencionado anteriormente por Graham (1988).

\section{AGRADECIMIENTOS}

Al Centro Amazónico de Educación Ambiental e Investigación (ACEER), y a su representante Aura Murrieta Torres por el financiamiento. A nuestro compañeros Nuri Pinedo, Cinthya Tuesta, Claudia Ríos y Roger Soplin, Gilmer Montero, Humberto Peña (Q.E.P.D) y Hugo Peña, por su apoyo en los trabajos de campo. A Paul Velazco, Sandra Velazco, Natalí Hurtado, Fanny Cornejo y a dos revisores anónimos por sus comentarios sobre el manuscrito. 


\section{REFERENCIAS}

Aguirre, L.F, Lens, L. \& Matthysen, E. (2003). Patterns of roost use by bats in a neotropical savanna: implications for conservation. Biological Conservation 111, 435-443

Aquino, R., Terrones, W., Cornejo, F. \& Heymann, E.W. (2008). Geographic distribution and possible taxonomic distinction of Callicebus torquatus populations (Pitheciidae: Primates) in Peruvian Amazonia. American Journal of Primatology 70, 1181-1186.

Aquino, R., Terrones, C., Navarro, R. \& Terrones, W. (2007). Evaluación del impacto de la caza en mamíferos de la cuenca del río Alto Itaya, Amazonía peruana. Revista Peruana de Biología 14(2), 181-186.

Aquino, R., Terrones, W., Navarro, R., Terrones, C. \& Cornejo, F. (2009). Caza y estado de conservación de primates en la cuenca del río Itaya, Loreto, Perú. Revista Peruana de Biología 15(2), 33- 39.

Ascorra, C.F. \& Wilson, D.E. (1992). Bat Frugivory and Seed Dispersal in the Amazon, Loreto, Perú. Publicaciones del Museo Historia Natural 'Javier Prado' Serie A Zoología 43, 1- 6.

Ascorra, C.F., Gorchov, D.L. \& Cornejo, F. (1993). The bats from Jenaro Herrera, Loreto, Peru. Mammalia 57, 533-552.

Bradbury, J.W. (1977). Social organization and communication. In Biology of Bats. New York,USA: Academic Press

Bradbury, J.W., \& Emmons, L.H. (1974). Social organization of some Trinidad bats. I. Emballonuridae. Zeitschrift für Tierpsychologie 36,137-83.

Brosset A. \& Charles-Dominique, P. (1991). The bats from French Guiana: A taxonomic, faunistic and ecological approach. Mammalia 54, 509 - 560.

Carvalho, A.L. de. (1940). Zur Biologie einer Fledermaus (Thyroptera tricolor Spix) des Amazonas. Sitzungsber. Gesells. Naturforsch. Freunde Berlin. 1939, 249-253.

Davis W.B. \& Dixon, J.R. (1976). Activity of Bats in a Small Village clearing near lquitos, Peru Journal of Mammalogy 57(4), 747-749

Dechmann, D.K.N., Kalko, E.K.V. \& Kerth, G. (2004). Ecology of an exceptional roost: Energetic benefits could explain why the bat Lophostoma silvicolum roosts in active termite nests. Evolutionary Ecology Research 6, 1037-1050.

Dechmann, D.K.N., Önig, B.K. \& Kerth, G. (2005). Mating system of aNeotropical roost making bat: The white-throated, round-eared bat, Lophostoma silvicolum (Chiroptera: Phyllostomidae). Behavioral Ecology and Sociobiology 58, 316-325.

Dinerstein, E., Olson, D.M., Graham, D.J., Webster, A.L., Primm, S.A., Bookbinder, M.P. \& Ledec, G. (1995). Una Evaluación del Estado de Conservación de las Ecorregiones de América Latina y el Caribe. Washington D.C., USA: Publicaciones Banco Mundial- Fondo Mundial para la Naturaleza.

Encarnación, F. (1993). El bosque y las formaciones vegetales en la llanura amazónica del Perú. Alma Máter 6, 95 - 114.
Fenton, M.B., Vonhof, M.J., Bouchard, S., Gill, S.A., Johnston, D.S., Reid, F.A., Riskin, D.K., Standing, K.L., Taylor, J.R. \& Wagner, R. (2000). Roosts used by Sturnira lilium (Chiroptera: Phyllostomidae) in Belize. Biotropica 32, 729-733.

Fenton, M.B., Bernard, E., Bouchard, E., Hollis, L., Johnston, D.S., Lausen, C.L., Ratcliffe, J.M., Riskin, D.K., Taylor, J.R. \& Zigouris, J. (2001). The bat fauna of Lamanai, Belize: roosts and trophic roles. Journal of Tropical Ecology 17, 511-524

Fleck, D.W., Voss, R.S., \& Simmons, N.B. (2002). Underdifferentiated taxa and sublexical categorization: an example from Matses classification of bats. Journal of Ethnobiology 22, 61-102.

Fleming T.H. \& Eby, P. (2003). Ecology of Bat Migration. In: Bat ecology. Chicago, USA: University of Chicago Press.

Foster M.S. (1992). Tent Roosts of Macconnell's Bat (Vampyressa macconnelli). Biotropica 24(3), 447-454

Gaisler. J. (1979). Ecology of bats. In: Ecology of small mammals. London. U.K.: Chapman \& Hall.

Giannini, N., \& Kalko, E.K.V. (2004). Trophic structure in a large assemblage of phyllostomid bats in Panama. Oikos 105, 209-220

Goodwin, G.G. \& Greenhall, A.M. (1961). A review of the bats of Trinidad and Tobago, descriptions, rabies infection, and ecology. Bulletin of the American Museum of Natural History $122,187-302$.

Graham, G. (1988). Interspecific associations among Peruvian bats at diurnal roosts and roost sites. Journal of Mammalogy 69, 711-720.

Greenhall, A.M. \& Paradiso, J.L. (1968). Bats and bat banding. Resource Publications. Sport Fish and Wildlife 72, 1- 48.

Handley, C.O. Jr. (1976). Mammals of the Smithsonian Venezuelan Project. Brigham Young University Science Bulletin, Biology Serie 20(5), 1- 89.

Hice, C.L., Velazco, P.M. \& Willig, M. (2004). Bats of the National Reserve Allpahuayo-Mishana, northeastern Peru, with notes on community structure. Acta Chiropterologica 6(2), 319-334.

Kalko, E.K.V., Ueberschaer, K. \& Dechmann, D. (2006). Roost Structure, Modification, and Availability in the White-throated Round-eared Bat, Lophostoma silvicolum (Phyllostomidae) Living in Active Termite Nests. Biotropica. 38(3), 398-404.

Koepcke, J. (1984). "Blattzelte" als Schlafplatze der Fledermaus Ectophylla macconnelli (Thomas, 1901) (Phyllostomi dae) im tropischen Regenwald von Peru. Saugertierk Mitt. 31, 123-26.

Kunz, T.H. \& Lumsden, L.F. (2003). Ecology of cavity and foliage roosting bats. In:T.H. Bat ecology. Chicago,USA: University of Chicago Press.

Kunz, T.H. (1982). Roosting ecology of bats. In: Ecology of bats. New York, USA: Plenum Press. 
LaVal, R.K. \& Rodríguez-H, B. (2002). Murciélagos de Costa Rica. Santo Domingo de Heredia, Costa Rica: INBio.

Lemke, T.O., Cadena, A., Pine, R.H. \& Hernández-Camacho, J. (1982). Notes on opossums, bats, and rodents new to the fauna of Colombia. Mammalia 46, 225-34.

Lim B.K., Engstrom, M.D., Reid, F.A., Simmons, N.B., Voss, R.S. \& Fleck, D.W. (2010). New species of Peropteryx (Chiroptera: Emballonuridae) from western Amazonia with comments on phylogenetic relationships within the Genus. American Museum Novitates 3686, 1-20.

López-Wong, C. (2002). Uso de hábitat por quirópteros en la Zona Reservada de Allpahuayo Mishana, Loreto-Perú. Tesis para optar título profesional de Biólogo. Loreto Perú: Universidad Nacional de la Amazonía Peruana.

Malleux M.O. (1982.) Inventarios forestales en bosques tropicales. Lima: Universidad Nacional Agraria La Molina.

McCarthy, T.J., Cadena, A.G. \& Lemke, T.O. (1983). Comments on the first Tonatia carrikeri (Chiroptera: Phyllostomatidae) from Colombia. Lozania. Acta Zoologica de Colombia 40, 1-6.

Pacheco, V., Cadenillas, R., Salas, E., Tello, C. \& Zeballos, H. (2009). Diversidad y endemismo de los mamíferos del Perú. Revista Peruana de Biología 16(1), 5-32

Rodríguez-Herrera, B., Medellín, R.A. \& Timm, R.M. (2007). Murciélagos neotropicales que acampan en hojas/ Neotropical tent-roosting bats. Heredia, Costa Rica: Editorial Inbio

Sanborn, C.C. (1937). American bats of the subfamily Emballonurinae. Field Museum Natural History, Zoology Series 20, 321-354.

Sanborn, C.C. (1949). Mammals from the Rio Ucayali. Peruvian Journal of Mammalogy 30, 277-88.

Sanborn, C.C. (1951). Mammals from Marcapata, southeastern Perú. Publicaciones del Museo Historia Natural Javier Prado' Serie A Zoología 6, 1-26.
Simmons, N.B., Voss, R.S. \& Fleck, D.W. (2002). A New Amazonian species of Micronycteris (Chiroptera: Phyllostomidae) with notes on the roosting behavior of sympatric congeners. American Museum Novitates 3358, 1-14

Simmons, N.B. \& Voss, R.S. (1998). The mammals of Paracou, French Guiana: a Neotropical lowland rain forest fauna. Part 1. Bats. Bulletin American Museum of Natural History 237, 1-219.

Simmons, N. (2005). Order Chiroptera. In: Mammals species of the world: a taxonomic and geographic reference. Volumen 1. Baltimore, USA: The Hopkins University.

Sioli, H. (1984). The Amazon and its main affluents: hydrography, morphology of the river courses and river types. In: The Amazon. Limnology and landscape ecology of a mighty tropical river and its basin. Dordrecht, The Netherlands: DrW. Junk Publishers.

Tello, J.G. \& Velazco, P.M. (2003). First description of a tent used by Platyrrhinus helleri (Chiroptera: Phyllostomidae). Acta Chiropterologica 5(2), 269-276.

Tuttle, M.D. (1970). Distribution and zoogeography of Peruvian bats, with comments on natural history. University of Kansas Science Bulletin 49:45-86.

Tuttle, M.D. 1976. Collecting techniques. In Biology of bats of the new world family Phyllostomatidae. Part I, Special Publications of the Museum 10. Lubbock, USA: Texas Tech University Press.

Velazco, P.M. \& Cadenillas, R. (2011). On the identity of Lophostoma silvicolum occidentalis (Davis \& Carter, 1978) (Chiroptera: Phyllostomidae). Zootaxa 2962, 1-20.

Voss, S.R.\& Emmons, L.H. (1996). Mammalian diversity in Neotropical lowland Rainforests: a preliminary Assessment. Bulletin of the American Museum of Natural History 230, $1-115$. 